\title{
Knee Range of Motion as a Discriminatory Tool Indicating Potential Meniscal Tears
}

\author{
James Hollier, MD, ${ }^{1}$ Claudia Leonardi, PhD, ${ }^{2}$ Linus Igbokwe, BS, ${ }^{3}$ Vinod Dasa, MD ${ }^{4}$ \\ ${ }^{1}$ School of Medicine, Louisiana State University Health Sciences Center, New Orleans, LA ${ }^{2}$ Department of Behavioral and Community \\ Health Sciences, School of Public Health, Louisiana State University Health Sciences Center, New Orleans, LA ${ }^{3}$ Robert Wood Johnson \\ Medical School, Rutgers, Piscataway, NJ ${ }^{4}$ Department of Orthopedics, School of Medicine, Louisiana State University Health Sciences Center, \\ New Orleans, LA
}

Background: Primary care physicians often encounter patients with knee pain and are faced with the dilemma of whether to refer patients to a specialist. Meniscal tears are the most common intraarticular knee injury but are challenging to accurately diagnose because of a lack of quantitative, accurate, and easy-to-administer tests. We conducted a retrospective medical record review to evaluate whether measurement of knee range of motion (ROM) via goniometry could discriminate between healthy and meniscus-altered knees.

Methods: A total of 110 adult patients met the inclusion criteria: age $\geq 18$ years; no history of contralateral knee pain, injury, or surgery; ROM data collected using a goniometer on both knees at the time of diagnosis; and a confirmed diagnosis of meniscus tear via magnetic resonance imaging. The following variables were obtained from medical records: age, sex, body mass index (BMI), ROM for both knees, surgical treatment, insurance coverage, Ahlbäck x-ray grades, Western Ontario and McMaster Universities Osteoarthritis Index (WOMAC), and the Oxford Knee Score (OKS).

Results: The majority of patients (96.4\%) exhibited $\mathrm{a} \geq 10^{\circ}$ difference in flexion between asymptomatic and symptomatic knees. No significant relationships were observed between age, BMI, and the decision to undergo surgery and the difference in flexion or extension ROM. Both the WOMAC and the OKS were significantly correlated with the degree of loss of flexion ROM.

Conclusion: The results suggest that knee flexion ROM may be a valuable tool for determining which patients presenting with new-onset ipsilateral knee pain should be referred to a specialist. Further investigation to determine the reliability and accuracy of knee ROM as a screening measure is warranted.

Keywords: Arthrometry-articular, diagnosis, range of motion-articular, referral and consultation, tibial meniscus injuries

Address correspondence to Vinod Dasa, MD, Department of Orthopedics, School of Medicine, Louisiana State University Health Sciences Center, 1542 Tulane Ave., Box-7, New Orleans, LA 70112. Tel: (504) 412-1700. Email: vdasa@lsuhsc.edu

\section{INTRODUCTION}

Meniscal tears are the most common intraarticular lesions of the knee. ${ }^{1}$ Arthroscopic partial meniscectomy is frequently used to surgically repair damaged areas to relieve pain and mechanical symptoms. National Survey of Ambulatory Surgery data show that the rate of knee arthroscopy in the United States increased by $49 \%$ from 1996-2006. ${ }^{2}$ Young athletes tend to experience traumatic tears during sports, while the middle-aged and elderly tend to experience a slow process of meniscal tissue degeneration. Among males aged $70-90$ years, the prevalence of meniscal tears is $>50 \%$, while in females aged $50-59$ years, the prevalence is $16 \%{ }^{3}$

The orthopedic patient population is expected to increase by $20 \%-45 \%$ by 2020 , while the number of orthopedic surgeons is expected to increase by only $2 \%$, creating a shortage of surgeons qualified to treat this growing population. ${ }^{4}$ The increase in demand for surgery and the shortage of or- thopedic specialists require the development of easily administered screening tools to increase efficiency in the management of patients with operable meniscus pathology. Currently, no standard criteria exist for referring primary care patients who are experiencing intraarticular knee pain to specialists. ${ }^{5-7}$ Consequently, orthopedic surgeons are largely responsible for determining whether patients have operable vs manageable knee pain, reducing the time they have available for surgery.

In the clinic setting, the diagnosis of a torn meniscus requires the assessment of a constellation of clinical signs using physical examination maneuvers and magnetic resonance imaging (MRI). However, research indicates that clinical signs have a variable predictive value in diagnosing meniscus tears, ${ }^{5,8-11}$ and the use of clinical maneuvers vs MRI is controversial. ${ }^{3,5,9,11,12}$ Simple assessments (eg, measurement of knee flexion) that can be reliably performed 
may help primary care physicians (PCPs) determine whether to refer patients with suspected meniscus tears. ${ }^{5,13}$ Comparing the ROM measurements of the affected and healthy knees could serve as a simple, easy-to-perform, quantitative screening to help PCPs manage patients with a suspected meniscus tear.

We designed this study to address 4 questions: (1) Does ROM differ between the affected and healthy knees in patients with diagnosed meniscus tears? (2) Does the difference in ROM between the affected and healthy knees change depending on patient characteristics? (3) Do patients who choose to undergo knee surgery exhibit any significant difference in ROM between the affected and healthy knees?

(4) Can we identify a flexion and/or extension value that best differentiates between the affected and healthy knees?

\section{METHODS}

The Louisiana State University Health Sciences Center (LSUHSC) Institutional Review Board approved this retrospective study. To identify eligible patients, we queried the LSUHSC healthcare network database for encounters with a diagnosis of meniscus tear (ICD-9 codes 717.2, 717.3, 717.4, 717.42, 717.49, and 836.1) between May 2007 and August 2013. Inclusion criteria were age $\geq 18$ years; MRIconfirmed diagnosis of ipsilateral meniscus tear; no recorded history of contralateral knee pain, injury, or surgery; and ROM data for both knees collected at the time of diagnosis.

A total of 1,960 encounters had a meniscus tear diagnosis in the specified time frame, of which 1,377 encounters were associated with a provider who recorded preoperative ROM measurements. From these encounters, we identified 685 unique events. Five events were excluded because of patient age $<18$ years, resulting in 680 eligible events. A total of 251 unique events had an associated MRI. A total of 110 unique patients met the remaining inclusion criteria of MRIconfirmed diagnosis of ipsilateral meniscus tear; no recorded history of contralateral knee pain, injury, or surgery; and ROM data for both knees collected at the time of diagnosis.

A goniometer was used to determine ROM while patients were actively flexing and extending both of their knees. Flexion and extension measurements were performed as described by Brosseau et al. ${ }^{14}$ The following variables were obtained from patients' medical records: age, sex, body mass index (BMI), election to have surgery, insurance coverage, Ahlbäck x-ray grades, Western Ontario and McMaster Universities Osteoarthritis Index (WOMAC), and the Oxford Knee Score (OKS).

The Ahlbäck classification scale has 6 grades: grade 0 (no radiographic sign of arthritis), grade 1 (joint space narrowing $<3 \mathrm{~mm}$ ), grade 2 (joint space obliteration), grade 3 (minor bone attrition $0-5 \mathrm{~mm}$ ), grade 4 (moderate bone attrition $>5-10 \mathrm{~mm}$ ), and grade 5 (severe bone attrition $>10 \mathrm{~mm}$ ). The WOMAC is a 24-item patient-reported outcome questionnaire used to assess pain, stiffness, and physical function in patients with hip and/or knee osteoarthritis on a 5-point Likert scale. A higher WOMAC score indicates worsening pain, stiffness, and functional limitations. ${ }^{15}$ The OKS was originally designed for inpatient use to assess the patient's perspective of outcomes following total knee arthroplasty, but its use has been validated as a reliable measurement for outpatients with osteoarthritis. ${ }^{16}$ The OKS is a 12 -item survey that assesses knee pain and physical disabi- lilty with a 5-point Likert scale. A higher OKS indicates worse knee pain and function.

Data were analyzed using SAS/STAT software v.9.2 (SAS Institute, Inc.). Differences in flexion (DF) and extension (DE) between symptomatic and asymptomatic knees were calculated for each patient as the degrees for the symptomatic knee minus the degrees for the asymptomatic knee. We used the Wilcoxon signed rank test to determine whether median DF and DE values were significantly different from 0 ; the Wilcoxon Mann-Whitney test to compare median DF and DE scores of patients who elected to have surgery and patients who decided not to undergo surgery; and the Spearman rank correlation coefficient to examine the relationships between DF/DE and patient age, BMI, WOMAC score, and OKS. To determine ROM levels that best differentiated between the symptomatic and asymptomatic knee, we constructed binary variables for flexion and extension using various cutoff values. A receiver operating characteristic analysis was conducted using the logistic procedure, and the optimum cutoff values for flexion and extension were chosen so that they produced the greatest area under the curve (AUC) calculated using the trapezoidal rule. Sensitivity, specificity, positive predictive value, negative predictive value, accuracy, likelihood ratios for positive and negative test estimates, and 95\% confidence intervals (Cls) were obtained using the frequency and GENMOD procedures. We considered 2 -tailed $P$ values $<0.05$ statistically significant.

\section{RESULTS}

Table 1 presents the characteristics of the study sample. The sample was predominantly female $(68.2 \%)$, had a mean age of 54.5 years (range, 23-79 years), and had a mean BMl of $33.6 \mathrm{~kg} / \mathrm{m}^{2}$ (range, $21.2-58.7 \mathrm{~kg} / \mathrm{m}^{2}$ ). At the time of diagnosis, all patients were evaluated with weightbearing anteroposterior analog printed knee x-rays; however, by the time of classification, we were unable to locate the printed x-rays for 9 patients. X-rays were available for 101 of the 110 patients, so those 101 patients could be diagnosed with no to mild osteoarthritis using the Ahlbäck x-ray classification system. Most patients (63.6\%) opted to undergo surgical treatment for their torn meniscus.

Flexion and extension measurements are reported in Table 2. Flexion of the asymptomatic knees ranged from $95^{\circ}-140^{\circ}$, with a median of $125^{\circ}$. In contrast, flexion of the symptomatic knees was considerably lower, ranging from $45^{\circ}-130^{\circ}$, with a median of $105^{\circ}$. The distribution of flexion measurements for symptomatic and asymptomatic knees is displayed in the Figure, A. The median DF of $-20^{\circ}$ (range, $-50^{\circ}-0^{\circ}$ ), calculated as the differences in degrees of the symptomatic knees minus the asymptomatic knees, was significantly different from zero $(P<0.0001)$. Most patients $\left(n=106 ; 96.4 \%\right.$ ) exhibited a reduction $\geq 10^{\circ}$ in flexion between asymptomatic and symptomatic knees, and 88 patients $(80 \%)$ exhibited a reduction $\geq 15^{\circ}$ in flexion between the asymptomatic and symptomatic knees (Figure, B).

Extension ranged from $-5^{\circ}-10^{\circ}$, with a median of $0^{\circ}$ for the asymptomatic knee and from $-5^{\circ}-30^{\circ}$ for the symptomatic knee, with a median of $5^{\circ}$. The distribution of extension measurements for symptomatic and asymptomatic knees is displayed in the Figure, $\mathrm{C}$. The median DE of $5^{\circ}$ (range, $-10^{\circ}-30^{\circ}$ ), calculated as the differences in degrees of the symptomatic knee minus the asymptomatic knees, was 
Table 1. Study Participant Characteristics

\begin{tabular}{lc}
\hline Variable & Value $\mathbf{n}=\mathbf{1 1 0}$ \\
\hline Age, years, mean \pm SD (range) & $54.5 \pm 12.4(23-79)$ \\
$\mathrm{BMI}, \mathrm{kg} / \mathrm{m}^{2}$, mean \pm SD (range) & $33.6 \pm 7.2(21.2-58.7)$ \\
Sex & $75(68.2)$ \\
Female & $35(31.8)$ \\
Male & \\
Ahlbäck classification ${ }^{\mathrm{a}}$ & $73(72.3)$ \\
0 & $27(26.7)$ \\
1 & $1(1.0)$ \\
2 & \\
Surgery & $70(63.6)$ \\
Yes & $40(36.4)$ \\
No & \\
Insurance type & $41(37.3)$ \\
Private & $32(29.1)$ \\
Medicaid & $37(33.6)$ \\
Medicare & \\
\hline
\end{tabular}

The Ahlbäck classification scale has 6 grades: grade 0 (no radiographic sign of arthritis), grade 1 (joint space narrowing $<3 \mathrm{~mm}$ ), grade 2 (joint space obliteration), grade 3 (minor bone attrition $0-5 \mathrm{~mm}$ ), grade 4 (moderate bone attrition $>5-10 \mathrm{~mm}$ ), and grade 5 (severe bone attrition $>10 \mathrm{~mm}$ ). The printed x-rays of 9 patients could not be located at the time of classification.

Note: Data are presented as n (\%) unless otherwise specified.
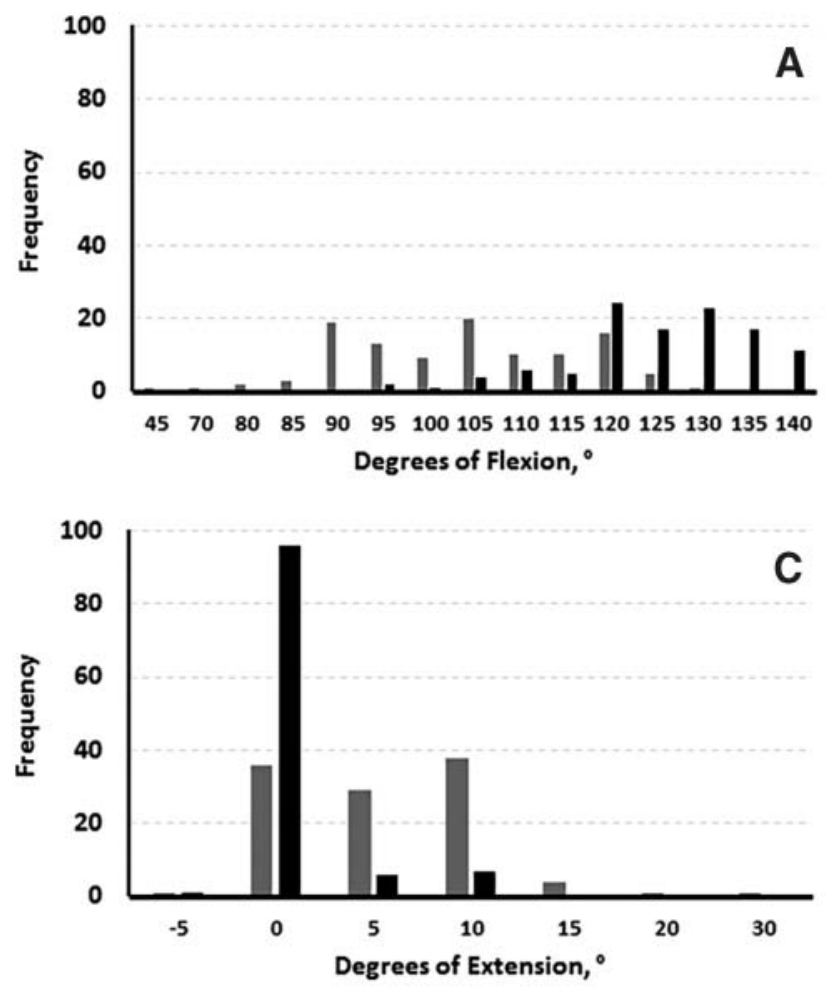

significantly different from zero $(P<0.0001)$. Sixty-five patients $(59.1 \%)$ exhibited a $D E \geq 5^{\circ}, 41$ patients $(37.3 \%)$ had a $0^{\circ}$ difference, and 4 patients (3.6\%) had a negative difference in the symptomatic knee (Figure, D).

Table 3 reports the difference in degrees of flexion and extension in patients who chose to undergo surgery vs patients who did not have surgery. We found no significant difference in DF $(P=0.549)$ or $\mathrm{DE}(P=0.413)$ between the 2 groups.

Table 4 reports the relationships between patient age, BMI, WOMAC score, and OKS and the DF and DE between the symptomatic and asymptomatic knees. We found no significant relationship between age or BMI and DF or DE. Both the WOMAC score $(r=0.363, P=0.0002)$ and the OKS ( $r=0.339, P=0.0004$ ) were significantly positively correlated with DF, indicating more impaired function and greater pain in patients with a larger DF between their asymptomatic and symptomatic knees. The OKS, but not the WOMAC score, was significantly positively correlated with DE. The statistically significant correlations between WOMAC or OKS scores and DF or DE were maintained after adjusting for sex, age, and BMI.

As stated in the Methods section, the optimal cutoff values for flexion and extension were selected so that the optimum combination of sensitivity and specificity was attained, as well as the highest likelihood ratio. The optimum levels of flexion and extension that provide the best discrimination points between symptomatic and asymptomatic knees were $120^{\circ}$ for flexion ( $A \cup C=81.8 \%$ ) and $5^{\circ}$ for extension ( $A U C=77.5 \%$ ).

Diagnostic parameters calculated for the chosen cutoff points are reported in Table 5 . Using a cutoff value of $120^{\circ}$
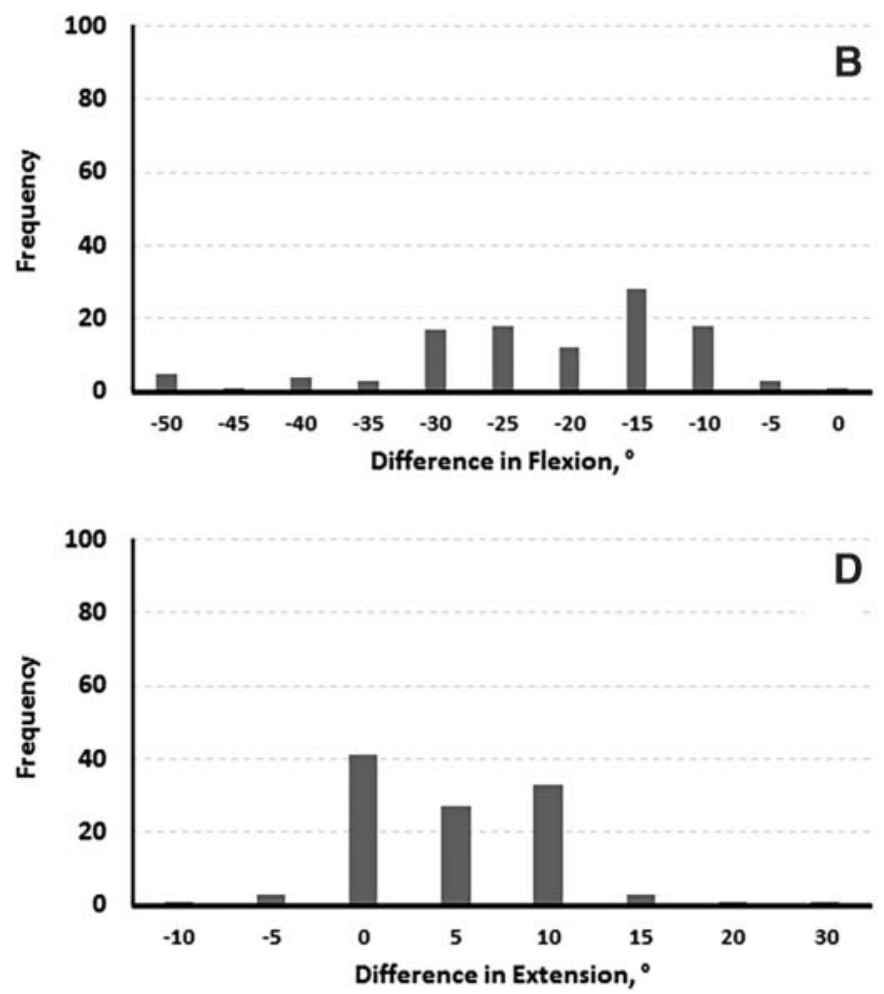

Figure. Distribution of bilateral knee flexion (A) for symptomatic (light grey) and asymptomatic (black) knees and their difference (B) and distribution of bilateral knee extension (C) for symptomatic (light grey) and asymptomatic (black) knees and their difference (D). The symptomatic ranges for degrees of flexion and extension range from $45^{\circ}-130^{\circ}$ and from $-5^{\circ}-30^{\circ}$, respectively. Frequencies at the tails of the symptomatic range are low and may be difficult to discern from the graph. 
Table 2. Median Flexion and Extension Differences in Symptomatic and Asymptomatic Knees in Patients With Ipsilateral Meniscus Tears $(\mathbf{n}=110)$

\begin{tabular}{lccc}
\hline Variable & Median & Range & P Value \\
\hline Flexion & & & \\
Symptomatic & $105^{\circ}$ & $45^{\circ}$ to $130^{\circ}$ & \\
Asymptomatic & $125^{\circ}$ & $95^{\circ}$ to $140^{\circ}$ & \\
Difference $^{\mathrm{a}}$ & $-20^{\circ}$ & $-50^{\circ}$ to $0^{\circ}$ & $<0.0001^{\mathrm{b}}$ \\
Extension & & & \\
Symptomatic & $5^{\circ}$ & $-5^{\circ}$ to $30^{\circ}$ & \\
Asymptomatic & $0^{\circ}$ & $-5^{\circ}$ to $10^{\circ}$ & \\
Difference $^{\mathrm{a}}$ & $5^{\circ}$ & $-10^{\circ}$ to $30^{\circ}$ & $<0.0001^{\mathrm{b}}$ \\
\hline
\end{tabular}

${ }^{a}$ Differences in flexion and extension were calculated for each patient as the degrees for the symptomatic knee minus the degrees for the asymptomatic knee.

${ }^{\mathrm{b}}$ Significantly different from 0 .

for flexion resulted in an $80 \%$ sensitivity and an $83.6 \%$ specificity, with predictive values and accuracy slightly above $80 \%$. Alternatively, the optimum extension cutoff value of $5^{\circ}$ resulted in very low sensitivity (33.0\%), specificity (11.9\%), accuracy $(22.5 \%)$, and predictive values.

\section{DISCUSSION}

Historically, methods for evaluating suspected meniscus tears, which are largely qualitative in nature, have shown variable sensitivity and specificity. ${ }^{9,11,13}$ Consequently, no widely accepted standard or uniform guideline exists to support decision-making regarding whether to refer patients with suspected meniscus tears to specialists. We found significant differences in DF and DE between asymptomatic and symptomatic knees in a cohort of patients with a confirmed diagnosis of meniscus tear, with the largest differences evident with flexion ROM; $96.4 \%$ of patients exhibited $a \geq 10^{\circ}$ difference in flexion ROM. These results suggest that knee $\mathrm{ROM}$, in combination with clinical maneuvers, could serve as a simple, low-cost, quantitative test to help determine whether PCPs should refer patients with a suspected meniscus tear for evaluation by an orthopedic surgeon.

The superiority of surgical vs conservative management of meniscus tears remains unclear. A longitudinal cohort study found that patients who received conservative, nonoperative management of medial meniscus tears experienced symptomatic and functional improvement during a 12-month follow-up period. ${ }^{17}$ Conversely, other studies have found no significant differences in outcomes after 3 years in patients with meniscus tears treated conservatively vs patients who underwent surgery. ${ }^{10,12}$ In our study, although patientreported function and pain were positively correlated with reduced flexion and extension ROM, suggesting that loss of
Table 4. Spearman Rank Correlation Between Differences in Flexion and Extension and Age, Body Mass Index (BMI), and Patient-Reported Outcomes

\begin{tabular}{|c|c|c|c|c|c|c|}
\hline \multirow[b]{2}{*}{ Variable } & \multicolumn{3}{|c|}{ Flexion Difference } & \multicolumn{3}{|c|}{ Extension Difference } \\
\hline & $\mathbf{n}$ & Rho ${ }^{a}$ & $P$ Value & $\mathbf{n}$ & Rho $^{\mathbf{a}}$ & $P$ Value \\
\hline Age, years & 110 & 0.082 & 0.393 & 110 & -0.042 & 0.662 \\
\hline BMI, $\mathrm{kg} / \mathrm{m}^{2}$ & 110 & 0.026 & 0.786 & 110 & 0.020 & 0.835 \\
\hline WOMAC score & 98 & 0.363 & 0.0002 & 98 & -0.187 & 0.065 \\
\hline Oxford Knee Score & 105 & 0.339 & 0.0004 & 105 & -0.235 & 0.016 \\
\hline WOMAC score ${ }^{b}$ & 98 & 0.345 & 0.0006 & 98 & -0.218 & 0.034 \\
\hline Oxford Knee Score ${ }^{b}$ & 105 & 0.385 & 0.0001 & 105 & -0.276 & 0.007 \\
\hline
\end{tabular}

a Spearman rank correlation coefficient.

${ }^{b}$ Spearman rank correlation adjusted for sex, age, and BMI.

WOMAC, Western Ontario and McMaster Universities Osteoarthritis Index.

ROM is associated with greater functional limitations, we found no apparent relationship between ROM and patients' decision to undergo surgical meniscus repair, suggesting that this decision is influenced by other factors.

This study had several limitations. First, although arthroscopy is the gold standard for diagnosing a meniscus tear, ${ }^{8,13}$ the present study used MRI to confirm a diagnosis of meniscus tear, as arthroscopy would have confounded our ability to examine the relationship between ROM and patients' decisions to undergo surgical repair. Second, our findings may have limited generalizability to the broader population, as the study sample was limited to patients who had a contralateral knee free from injury, pain, and surgery. In addition, only a small portion of eligible patients had ROM measured in both knees. Last, although some evidence suggests that race may be significantly related to knee $\mathrm{ROM},{ }^{18}$ this analysis did not examine the relationship between race and knee ROM because of the small sample size available retrospectively.

\section{CONCLUSION}

Because the number of patients requiring evaluation for meniscus tears is expected to increase dramatically and the current methods for diagnosing meniscus tears have limitations, a simple and accurate screening tool to help generalist physicians determine whether to refer patients with suspected meniscus tears to a specialist is needed. Our results indicate that measurement of knee ROM with a goniometer could be an easily implemented, noninvasive, and inexpensive means of assessing patients presenting with new onset ipsilateral knee pain with a suspicion of significant intraarticular pathology. Further research is needed to determine optimal thresholds for loss of ROM that warrant referral and to determine the sensitivity and specificity of knee ROM as a screening tool for diagnosing meniscus tears.

Table 3. Flexion and Extension Difference in Patients Who Had Surgery vs Patients Who Did Not $(n=110)$

\begin{tabular}{lccc}
\hline Variable & Surgery Group & No-Surgery Group & $\boldsymbol{P}$ Value \\
\hline Flexion difference & $-20^{\circ}\left(-50^{\circ}\right.$ to $\left.0^{\circ}\right)$ & $-15^{\circ}\left(-50^{\circ}\right.$ to $\left.-10^{\circ}\right)$ & 0.549 \\
Extension difference & $5^{\circ}\left(-5^{\circ}\right.$ to $\left.30^{\circ}\right)$ & $5^{\circ}\left(-10^{\circ}\right.$ to $\left.20^{\circ}\right)$ & 0.413 \\
\hline
\end{tabular}

Note: Data are reported as median (range). 
Table 5. Diagnostic Parameters for Using Flexion or Extension to Identify a Meniscus Tear

\begin{tabular}{|c|c|c|c|c|}
\hline \multirow[b]{2}{*}{ Item } & \multicolumn{2}{|c|}{ Flexion $^{a}$} & \multicolumn{2}{|c|}{ Extension $^{\mathbf{b}}$} \\
\hline & Estimate & $95 \% \mathrm{Cl}$ & Estimate & $95 \% \mathrm{Cl}$ \\
\hline Sensitivity, \% & 80.0 & $72.5-87.5$ & 33.0 & $24.2-41.9$ \\
\hline Specificity, \% & 83.6 & 76.7-90.6 & 11.9 & $5.8-18.0$ \\
\hline Positive predictive value, $\%$ & 83.0 & $75.9-90.2$ & 27.3 & 19.7-34.9 \\
\hline Negative predictive value, $\%$ & 80.7 & $73.5-88.0$ & 15.1 & $7.6-22.7$ \\
\hline Diagnostic accuracy, \% & 81.8 & 76.7-86.9 & 22.5 & $16.9-28.0$ \\
\hline Likelihood ratio of positive test & 4.9 & 2.6-7.1 & 0.38 & $0.27-0.49$ \\
\hline Likelihood ratio of negative test & 0.24 & $0.14-0.34$ & 5.6 & $2.5-8.8$ \\
\hline
\end{tabular}

$\mathrm{Cl}$, confidence interval.

${ }^{\mathrm{a}}$ Flexion cutoff for meniscus tear diagnosis was $120^{\circ}$.

${ }^{b}$ Extension cutoff for meniscus tear diagnosis was $5^{\circ}$.

\section{ACKNOWLEDGMENTS}

The authors have no financial or proprietary interest in the subject matter of this article.

The authors thank Amy Bronstone, PhD, for assistance in editing this paper.

\section{REFERENCES}

1. Mordecai SC, Al-Hadithy N, Ware HE, Gupte CM. Treatment of meniscal tears: an evidence based approach. World J Orthop. 2014 Jul 18;5(3):233-241. doi: 10.5312/wjo.v5.i3.233.

2. Kim S, Bosque J, Meehan JP, Jamali A, Marder R. Increase in outpatient knee arthroscopy in the United States: a comparison of National Surveys of Ambulatory Surgery, 1996 and 2006. J Bone Joint Surg Am. 2011 Jun 1;93(11):994-1000. doi: 10.2106/JBJS.I.01618.

3. Beaufils $P$, Becker $R$, Kopf $S$, et al. Surgical management of degenerative meniscus lesions: the 2016 ESSKA meniscus consensus. Knee Surg Sports Traumatol Arthrosc. 2017 Feb;25 (2):335-346. doi: 10.1007/s00167-016-4407-4.

4. Willams TE, Satiani B, Ellison C. The Coming Shortage of Surgeons: Why They Are Disappearing and What That Means for Our Health. Santa Barbara, CA: Praeger; 2009:69-71.

5. Pookarnjanamorakot $C$, Korsantirat T, Woratanarat P. Meniscal lesions in the anterior cruciate insufficient knee: the accuracy of clinical evaluation. J Med Assoc Thai. 2004 Jun;87(6):618-623.

6. Smith BE, Thacker D, Crewesmith A, Hall M. Special tests for assessing meniscal tears within the knee: a systematic review and meta-analysis. Evid Based Med. 2015 Jun;20(3):88-97. doi: 10.1136/ebmed-2014-110160.

7. Snoeker BA, Lindeboom R, Zwinderman AH, Vincken PW, Jansen JA, Lucas $C$. Detecting meniscal tears in primary care: reproducibility and accuracy of 2 weight-bearing tests and 1 non-weight-bearing test. J Orthop Sports Phys Ther. 2015 Sep;45 (9):693-702. doi: 10.2519/jospt.2015.5712.

8. Eren OT. The accuracy of joint line tenderness by physical examination in the diagnosis of meniscal tears. Arthroscopy. 2003 Oct;19(8):850-854.
9. Rose NE, Gold SM. A comparison of accuracy between clinical examination and magnetic resonance imaging in the diagnosis of meniscal and anterior cruciate ligament tears. Arthroscopy. 1996 Aug;12(4):398-405.

10. Yim JH, Seon JK, Song EK, et al. A comparative study of meniscectomy and nonoperative treatment for degenerative horizontal tears of the medial meniscus. Am J Sports Med. 2013 Jul;41(7):1565-1570. doi: 10.1177/0363546513488518.

11. Mohan BR, Gosal HS. Reliability of clinical diagnosis in meniscal tears. Int Orthop. 2007 Feb;31(1):57-60.

12. Rimington T, Mallik K, Evans D, Mroczek K, Reider B. A prospective study of the nonoperative treatment of degenerative meniscus tears. Orthopedics. 2009 Aug;32(8). doi: 10.3928/01477447-20090624-06.

13. Fowler PJ, Lubliner JA. The predictive value of five clinical signs in the evaluation of meniscal pathology. Arthroscopy. 1989;5 (3):184-186.

14. Brosseau $L$, Balmer $S$, Tousignant $M$, et al. Intra- and intertester reliability and criterion validity of the parallelogram and universal goniometers for measuring maximum active knee flexion and extension of patients with knee restrictions. Arch Phys Med Rehabil. 2001 Mar;82(3):396-402.

15. Western Ontario and McMaster Universities Osteoarthritis Index (WOMAC). American College of Rheumatology. https:/ /www.rheumatology.org/l-Am-A/Rheumatologist/Research /Clinician-Researchers/Western-Ontario-McMaster-Universities -Osteoarthritis-Index-WOMAC. Accessed November 30, 2017.

16. Xie F, Ye H, Zhang Y, Liu X, Lei T, Li SC. Extension from inpatients to outpatients: validity and reliability of the Oxford Knee Score in measuring health outcomes in patients with knee osteoarthritis. Int J Rheum Dis. 2011 May;14(2):206-10. doi: 10.1111/j.1756-185X.2010.01580.x.

17. Neogi DS, Kumar A, Rijal L, Yadav CS, Jaiman A, Nag HL. Role of nonoperative treatment in managing degenerative tears of the medial meniscus posterior root. J Orthop Traumatol. 2013 Sep;14(3):193-199. doi: 10.1007/s10195-013-0234-2.

18. Leszko F, Hovinga KR, Lerner AL, Komistek RD, Mahfouz MR. In vivo normal knee kinematics: is ethnicity or gender an influencing factor? Clin Orthop Relat Res. 2011 Jan;469(1):95106. doi: 10.1007/s11999-010-1517-z.

This article meets the Accreditation Council for Graduate Medical Education and the American Board of Medical Specialties Maintenance of Certification competencies for Patient Care, Medical Knowledge, and Practice-Based Learning and Improvement. 